\title{
VIEWPOINT
}

\section{The strange magnetism of oxides and carbons}

\author{
Marshall Stoneham \\ London Centre for Nanotechnology and Department of Physics and Astronomy, \\ University College London, WC1E 6BT, UK
}

Received 6 July 2009

Published 3 February 2010

Online at stacks.iop.org/JPhysCM/22/074211

\begin{abstract}
Magnetism is not usually expected in simple sp oxides like $\mathrm{MgO}$ or in carbons like graphite. Yet basic intrinsic defects in these systems can be magnetic in ways that seem to be shared by more complex oxides. A second puzzle comes from reports of possible room temperature ferromagnetism in simple oxides, where experiments are not always in agreement. This paper discusses what determines whether point defects like cation vacancies in oxides have magnetic or non-magnetic ground states. It also discusses the possible connections between point defect ground states and oxide ferromagnetism. The connectivity issue raises questions about possible diffuse states in nanocrystalline oxides, several possibilities being outlined. These ideas raise the further possibility that the magnetism might be written in these oxides at the nanoscale, perhaps using atomic force microscopy.
\end{abstract}

\section{Introduction}

The observation of ferromagnetism in thin films of $\mathrm{HfO}_{2}$ (Venkatesan et al 2004) stimulated much activity. The many subsequent reports of magnetism in simple oxides and carbons continue to be puzzling. Isolated paramagnetic dopants or defects are well known, of course. Ferromagnetic inclusions are well known. But ferromagnetism in closed-shell oxides, even ones considered to have only low atomic number elements with just s- and p-electrons, is odd. In this Viewpoint I want to highlight some of the explanations and some of their difficulties and how they might be resolved. I shall also put the new results in context with much older results involving what seems to be key physics. This context helps one to understand problems that arise with some common theoretical approaches based on standard electronic structure codes.

For simplicity, and without serious ambiguity, I shall refer to specific charge states of ions and to localized and delocalized states in the usual relatively informal way. Localized states are usually identified by spin resonance or some other spectroscopy. A localized state might be trapped or self-trapped, or both (see, e.g., Itoh and Stoneham 2001). Thus a hole might be trapped at a ferrous ion $\mathrm{Fe}^{2+}$ to form a ferric ion $\mathrm{Fe}^{3+}$, or electron(s) trapped at an oxide ion vacancy to form $\mathrm{F}^{+}$or $\mathrm{F}^{0}$ centres. A hole in $\mathrm{MgO}$ might be trapped at a cation $\left(\mathrm{Mg}^{2+}\right)$ vacancy and additionally self-trapped on one of the oxide ion neighbours, rather than delocalized over all six of them. Delocalized states are often identified in transport studies. In the highly ionic oxides, delocalized states are largely unknown, though there are fascinating states associated with $\mathrm{LaAlO}_{3} / \mathrm{SrTiO}_{3}$ interfaces that can even show superconductivity. The electron-phonon coupling in most ionic oxides is strong, leading to incoherent hopping with mobilities that rise with temperature but remain less than about $1 \mathrm{~cm}^{2} \mathrm{~V}^{-1} \mathrm{~s}^{-1}$ (essentially one hop every lattice vibration period). In non-polar semiconductors and many carbons, delocalized states exist and are readily populated; for them, the mobility falls with phonon scattering as temperature rises, to a mobility of order $1 \mathrm{~cm}^{2} \mathrm{~V}^{-1} \mathrm{~s}^{-1}$ when the mean free path is of the order of one interatomic spacing.

The key facts for oxides seem to be these. First, the ferromagnetism is found in a range of oxides, with different structures (including $\mathrm{MgO}, \mathrm{Al}_{2} \mathrm{O}_{3}, \mathrm{CeO}_{2}, \mathrm{HfO}_{2}$ and $\mathrm{TiO}_{2}$; also, with dilute transition metal doping, in $\mathrm{MgO}, \mathrm{ZnO}, \mathrm{CeO}_{2}, \mathrm{HfO}_{2}$, $\mathrm{TiO}_{2}, \mathrm{SnO}_{2}, \mathrm{In}_{2} \mathrm{O}_{3}$, indium tin oxide and $\left.(\mathrm{La}, \mathrm{Sr}) \mathrm{TiO}_{3}\right)$, as well as $\mathrm{CdS}$ and $\mathrm{GaN}$. These systems show different forms of disorder; $\mathrm{MgO}$, for instance, is usually highly stoichiometric, whereas $\mathrm{TiO}_{2}$ is often oxygen-deficient. Moreover, these systems include highly insulating cases for which the Fermi level is not rapidly established (transition metal ions in $\mathrm{MgO}$ can remain in the 'wrong' charge states for weeks, for instance). Evidently, we cannot assume some very special 
conjunction of state energies for all these systems. Second, the ferromagnetism seems clear in thin films and nanocrystals; evidence for the bulk is far less certain (Sundaresan et al 2006, Sundaresan and Rao 2009, Coey and Chambers 2008, Liu et al 2008, Hu et al 2008). Third, the ferromagnetism is seen for sparse populations of point defects or dopants. There may be no evident transition metal impurity and, even when there is a transition metal impurity, the concentration need not be high. Fourth, there is no evidence for occupied delocalized states in these oxides. Transport measurements for nanocrystals present problems, of course, and it would be wrong to assume an absence of relatively delocalized states.

There is, of course, no shortage of individual paramagnetic defects or impurities. In some especially interesting cases, there is either a magnetic ground state or a magnetic excited state that is populated at room temperature. But ferromagnetism requires something akin to a phase change, i.e. coupling to link many localized centres, if such are involved. The obvious couplings are relatively short range and do not seem to supply what would be needed. The suspicion must be that there are some relatively delocalized states of some sort. If so, what states are these? Is the magnetism entirely associated with delocalized states? If not, so localized states are involved, but how are they linked by these supposed delocalized states?

It is helpful to start by discussing possible localized states, partly to try to understand the magnetism, and partly to get to grips with some of the aspects that cause problems in standard theory. In particular, there must be concern for the relative energies of spin triplet and spin singlet states when the energy differences are small. Likewise, self-trapping or not will be an issue. There is also the continuing problem of delocalized bound states.

There are also two broader questions. First, apparently analogous ferromagnetism is seen in a range of carbons, often after irradiation, when vacancies are likely to be present (graphite, buckeyballs, carbon nanotubes, nanodiamond, carbon nanofoams, some conducting polymers). Is the mechanism the same in oxides and carbons? If so, there seems to be a novel class of systems showing s-p magnetism. The possible mechanisms I discuss in the following sections. Secondly, does understanding let us identify new and useful magnetic systems? There are, of course, many standard ferromagnets; what the new systems may have to offer will depend on how they might be integrated into novel devices or exploited in nanoscale experiments. As outlined later, there may be ways to write magnetism at the nanoscale.

\section{Cation vacancies in alkaline earth oxides: charge localization and the $\mathrm{V}^{-}$centre}

The extensive studies over many years of cation vacancies in simple oxides (like $\mathrm{MgO}, \mathrm{CaO}, \mathrm{SrO}, \mathrm{BeO}, \mathrm{ZnO}$ and $\mathrm{Al}_{2} \mathrm{O}_{3}$ ) have been reviewed by Schirmer (2006) and by Stoneham et al (2007). These papers, and also Stoneham (1975), likewise discuss related centres in which an alkali ( $\mathrm{Li}$ or $\mathrm{Na}$ ) replaces a divalent cation. The cation vacancy exists in several possible charge states. Thus the so-called $\mathrm{V}^{0}$ centre is equivalent to removing a metal atom and the $\mathrm{V}^{-}$ centre has one extra electron. In an ionic picture, with
$\mathrm{Mg}^{2+}$ and $\mathrm{O}^{2-}$ as closed-shell ions, $\mathrm{V}^{-}$would have one trapped hole and $\mathrm{V}^{0}$ would have two trapped holes (there are equivalent ionic and covalent descriptions, see Stoneham (1975) section 15.5.3; see also Catlow and Stoneham (1983)). These experimental studies showed several novel features confirmed by detailed theory. Thus, in the $\mathrm{MgO} \mathrm{V}^{-}$centre, spin resonance showed the hole localizes on one oxygen; in the simplest ionic picture, the cation vacancy has one $\mathrm{O}^{-}$ neighbour and five $\mathrm{O}^{2-}$ neighbours. Its optical transitions can be intrasite (at the $\mathrm{O}^{-}$) or intersite charge transfers. Even this simple defect raises important questions. Polarization energies favour localization of the hole onto one oxygen, whereas tunnelling energies favour delocalization. But one can estimate tunnelling energies from the observed splitting of the charge transfer energies and one can estimate polarization energies by tried and tested methods. Provided the calculation is good enough, the answers are consistent. 'Good enough' means ensuring that self-interactions are corrected in density functional theory and making sure that electronic and ionic polarizations are correctly assigned by avoiding simplistic one-coordinate models. This separation of polarization terms is especially clear for the $\mathrm{V}^{-}$centre in $\mathrm{ZnSe}$ (Harding and Stoneham 1982). If not done, the tunnelling parameters from observed charge transfer spectra imply that the hole should not localize on a single $\mathrm{Se}$, contrary to experiment. Many discussions of colossal magnetoresistance oxides fail to separate these terms and can be inconsistent in important ways.

The first test of theory is whether the hole localizes on one oxygen or spreads over all six neighbours. This involves a balance between a tunnelling energy, favouring the delocalized state, and a polarization energy, favouring a localized state. The shell model, a general model within the harmonic and dipole approximations, gives good estimates of the polarization energy. The tunnelling term can be estimated from observed spectra, as noted, or from full-scale calculations, though these calculations with standard density functional theory can have problems because of self-interaction terms. The systematic early calculations show that, in all cases tested, the hole(s) localize on single oxygen(s). Further, Hartree-Fock theory with embedding in a shell model works very well for spin resonance data, optical data, for the dipole moment (there are some issues concerning effective field corrections that need resolution), reorientation energies and data from trapping energetics. The same conclusion holds for related centres, e.g. $\mathrm{Na}$ or $\mathrm{K}$ at the $\mathrm{Mg}$ site, $\mathrm{Al}$ at the second-neighbour site or a second hole trapped to form a $\mathrm{V}^{0}$ centre.

A strong second test comes from the optical data. The intrasite transitions, essentially at one oxygen, give the transition energy needed in estimates of $g$ factors. Predictions of energy and oscillator strength made by combining HartreeFock theory with shell model embedding (Norgett et al 1977) agree well with observation. Charge transfers provide the second class of transition (Schirmer et al 1974), with the hole moving from one site to either a molecular orbital involving the four nearest oxygen neighbours or to the oxygen directly opposite on the other side of the vacancy. The energy difference between these two transitions for $\mathrm{MgO}$ allows an estimate of the tunnelling energy terms. The optical 
transition energies for these charge transfers are dominated by polarization energies, and allow one to check the shell model contributions. In short, the one-hole $\mathrm{V}^{-}$centre seems rather well understood.

\section{Cation vacancies in alkaline earth oxides: spin states}

Experimentally, there is evidence that the two holes (two $\mathrm{O}^{-}$ ions in the ionic picture) of the $\mathrm{V}^{0}$ centre localize on opposite sides of this $\mathrm{MgO}$ cation vacancy. For $\mathrm{MgO}$, the ground state has spin $S=0$, with a magnetic $(S=1)$ state a few meV above the non-magnetic ground state. Experiment shows the triplet state is the ground state for $\mathrm{BeO}$ (Maffeo et al 1970) and $\mathrm{Al}_{2} \mathrm{O}_{3}$ (Cox 1966, 1971, 1972) or close in energy to the nonmagnetic ground state in $\mathrm{MgO}$ (Wertz et al 1959, Rius and Cox 1974) and $\mathrm{CaO}$ (Henderson and Tomlinson 1969, Abraham et al 1975, Henderson 2006); data are inconclusive for $\mathrm{ZnO}$ (Galland and Herv'e 1970).

Naively, one might expect the singlet $S=0$ state to be lowest: for two electrons, a theorem due to Wigner and to Mermin requires the ground state to be a singlet (e.g. the $\mathrm{He}$ atom). But if there are only two interesting electrons (closed shells are not normally interesting), the ground state can be a triplet (e.g. Hund's rules for a $3 \mathrm{~d}^{2}$ system). One suggested mechanism for a triplet ground state in some (but not all) $\mathrm{V}^{0}$ centres was proposed by Stoneham (1975), Kollmar and Klein (1993) cite a similar idea for organics due to McConnell (1967).

For $\mathrm{MgO}$, the neutral cation vacancy has two holes trapped on oxygen neighbours. Stoneham suggested that the admixture of $\mathrm{O}^{2-} \mathrm{O}^{0}$ and $\mathrm{O}^{0} \mathrm{O}^{2-}$ states into the basic $\mathrm{O}^{-}-$ $\mathrm{O}^{-}$configuration was the key. Suppose the energies in the basic $\mathrm{O}^{-}-\mathrm{O}^{-}$configuration are $E_{\mathrm{S} 0}$ for the singlet and $E_{\mathrm{T} 0}$ for the triplet. Then the admixture will involve admixture matrix elements $\delta$ and the energy separations $\varepsilon$ of the $\mathrm{O}^{2-} \mathrm{O}^{0}$ and $\mathrm{O}^{0} \mathrm{O}^{2-}$ states. The energies, for a weak admixture, become $E_{\mathrm{T}}=E_{\mathrm{T} 0}-\delta_{\mathrm{T} 2}^{2} / \varepsilon_{\mathrm{T}}$ for the triplet state and $E_{\mathrm{S}}=E_{\mathrm{S} 0}-$ $\delta_{\mathrm{S} 2}^{2} / \varepsilon_{\mathrm{S}}$ for the singlet state. These energies were analysed in detail by Pathak et al (1976), combining unrestricted HartreeFock theory with shell model methods for the polarization and electric field aspects. Simple calculations (e.g. standard Hartree-Fock) will give $S=0$ as the ground state. But the admixture lowers the triplet more in energy (recall that free $\mathrm{O}^{0}$ has a triplet state lowest in energy and $\mathrm{O}^{2-}$ has a closed shell). The correction from these extra terms can be a few tens of meV, similar in magnitude but opposite in sign to the splitting without admixture. The competition between the two terms could lead to either the singlet or the triplet being lowest, depending on details, as apparently observed. In the case of $\mathrm{MgO}$, theory indeed correctly predicts the $S=1$ state to be higher in energy by a few tens of meV, but such success must be partly fortuitous. There is a very delicate balance of energies; thus recent careful density functional calculations for the $\mathrm{V}^{0}$ centre in $\mathrm{CaO}$ (Osorio-Guill'en et al 2006) predict a very stable ferromagnetic state, contrary to experiment.

\section{Spin states for systems with oxygen vacancies}

Measurements have suggested ferromagnetism in $\mathrm{HfO}_{2}$ (Coey et al 2005) have been controversial (Abraham et al 2005, Ramachandra Rao et al 1999, Hong et al 2006), though given some support from theory (Pemmajaru and Sanvito 2005) and from apparently similar magnetism in other oxides, like $\mathrm{CeO}_{2}$, $\mathrm{SnO}_{2}$ and $\mathrm{TiO}_{2}$, usually in circumstances suggesting oxygen vacancy involvement. The tantalizing $\mathrm{HfO}_{2}$ results suggest moments of one Bohr magneton for each few tens of molecular units, with useful magnetism at room temperature. Such behaviour could be the basis for new magnetic and spintronic devices.

For oxides with cations that can readily change valence, e.g. $\mathrm{TiO}_{2}$ or $\mathrm{HfO}_{2}$, one can see again how oxygen vacancies might have triplet ground states. If one oxide ion $\mathrm{O}^{2-}$ is removed from perfect $\mathrm{TiO}_{2}$, the cations would normally have an empty $d$ shell $\left(3 \mathrm{~d}^{0}\right)$. Now add an electron. If the charge localizes (this seems still unsettled, but there is some evidence for this from surface studies (Zhang et al 1991)), one closedshell $\mathrm{Ti}^{4+}$ neighbour may become $\mathrm{Ti}^{3+}$. A second added electron may localize on the same cation as the first, giving a $3 d^{2}$ ion for which Hund's rules imply a spin triplet state. Alternatively, the second electron could localize on a different cation neighbour to the oxygen vacancy, probably on the opposite side of the $\mathrm{O}$ vacancy to minimize Coulomb repulsion. If so, then, just as for the $\mathrm{V}^{0}$ centres in $\mathrm{MgO}$, there might be configuration admixture, lowering the $S=1$ state relative to the $S=0$ Hartree-Fock ground state. Again, there may be a delicate balance between singlet and triplet states that can either favour the triplet state or make it accessible at room temperature.

For oxygen vacancies in alkaline earth oxides, the oneelectron $\mathrm{F}^{+}$and two-electron $\mathrm{F}^{0}$ centres have been studied extensively, and there are good reasons for their different behaviour. These electrons have a substantial part of their charge within the vacancy region (an electron can spread into a void space, whereas the trapped holes for a cation vacancy cannot, of course) and the singlet $S=0$ state is always lowest. In essence, the tunnelling integral is substantial (several eV) and the polarization gain from localization is reduced because it is the relatively unpolarizable $\mathrm{Mg}$ ions, not the polarizable oxygens, that are most affected. If the electrons were excluded from the vacancy site, perhaps by a substitutional rare gas atom (like $\mathrm{Ar}$ at the $\mathrm{O}$ site in $\mathrm{MgO}$ ) or even a substitutional nitrogen atom, the situation would be more closely analogous to the cation vacancy $\mathrm{V}^{0}$ centre, and it is possible that a triplet $S=1$ state might be seen. But is the $\mathrm{Mg}^{0} \mathrm{Mg}^{2+}$ triplet lower in energy than the singlet? Possible not. But Ar and C implants of $\mathrm{ZnO}$ do create ferromagnetism (Zhou et al 2008, see also Ye et al 2008), and detailed calculations for $\mathrm{V}^{0}$ in $\mathrm{ZnO}$ could be rewarding.

The discussion above suggests two critical roles for electron correlations: first, in the localization on neighbours to the vacancy, and secondly in the configuration admixture that provides a significant part of the singlet-triplet splitting. These roles are critical for simple centres to have an occupied $S=1$ state at room temperature. The presence of such centres does not amount to a ferromagnet, but they are key ingredients. 


\section{The problem of connectivity}

Magnetism is unexpected in these robust and readily fabricated oxides and carbon-based systems and, if reproducible and controllable, might be highly desirable. We have argued that there is a respectable case for intrinsic defects, like vacancies, providing a population of spin triplet systems even at room temperature. But this would solve only one part of the problem. What are credible mechanisms that give sufficient linkage to create ferromagnetism at the relatively low concentrations involved?

Even if there is a crucial role for traces of magnetic impurities, the magnetism still appears at low concentrations. Ferromagnetism needs the magnetic components to be linked. In dilute random systems, there may not be a phase transition in the usual sense: the fluctuations in the field driving the transition may be much larger than the average value of that field; essentially, small clusters freeze out at varying temperatures (see, e.g., Stoneham and Bullough 1970). But it is hard to avoid some sort of percolation problem (OsorioGuill'en et al 2006). It does not suffice (as many authors imply) to predict paramagnetic defect centres. In some systems, there may be longer-range ferromagnetic interactions from partfilling of $3 \mathrm{~d}$ conduction band resonances, as suggested by Raebiger et al (2009). But many systems seem not to show ferromagnetism in the bulk.

One important clue must be that ferromagnetism is seen in thin films or nanoparticles, apparently not in bulk samples. What is special about the nanoscale? The answer is not clear, but two types of idea may help. First, Coey et al (2008) have suggested a charge transfer model. Defects in the system will give rise to a narrow peak in the local density of states, but normally the Fermi level will not lie within that peak. If there are dopants present that can exist in more than one charge state (a common situation), then electron transfer might lead to a Stoner splitting, hence spontaneous Stoner ferromagnetism in a connected defect-rich region, such as a nanoparticle surface. This idea is interesting. One concern is that the magnetism is seen in so many distinct oxides, and the Coey et al proposal seems to need rather specific conditions. There remains still the puzzle of what the nature of the connected region is. Coey et al (2005) suggested possible shallow donor electrons that form bound magnetic polarons, such that their overlap in a spin-split impurity band might provide the links, applying their ideas to $\mathrm{ZnO}, \mathrm{TiO}_{2}$ and $\mathrm{SnO}_{2}$. It may be relevant that, in insulating oxides like $\mathrm{MgO}$, a Fermi level is only established very slowly indeed: 'wrong' charge states of the transition metal ion can survive for weeks or months, and indeed the Elovich kinetics seen for $\mathrm{MgO}$ nanocrystals may suggest that charge transfer is not fast (Nelson and Tench 1967, Nelson et al 1968), so kinetics would be an issue. So it may not be appropriate to use equilibrium ideas for charge transfers.

What other options might there be? We seek a mechanism that operates in nanocrystals, and only in nanocrystals or near surfaces. Could the strongly localized paramagnetic states contain an admixture of some more extended state in nanocrystals? The extended component, which provides longer-range interactions, would not be detectable in the spin resonance of bulk crystals, but would emerge only in nanoscale systems. In essence, what is needed is a relatively delocalized mediating state. Just as ionic configurations $\left(\mathrm{O}^{2-} \mathrm{O}^{0}\right.$ and $\mathrm{O}^{0} \mathrm{O}^{2-}$ in simple sp oxides) enable a local ferromagnetic interaction at some cation vacancies, so the extended mediating state should enable more widespread ferromagnetic coupling. Like the $\left(\mathrm{O}^{2-} \mathrm{O}^{0}\right.$ and $\left.\mathrm{O}^{0} \mathrm{O}^{2-}\right)$ ionic configurations, such a delocalized mediating state would be essentially unoccupied in the ground state, merely weakly admixed. Just what could these delocalized components be? Three groups of ideas warrant consideration. First, there are ideas involving diffuse (extended, but possibly bound) states at external surfaces. Secondly, there are diffuse states at internal interfaces or at extended defects. Thirdly, whilst small polarons certainly play a role, there may be situations where the large polaron form is only slightly higher in energy, and could contribute to longerrange interactions.

As regards the last category, invoking small and large polarons, in $\mathrm{HfO}_{2}$, detailed calculations and experiment indicate (Muñoz Ramo et al 2007) that paramagnetic negatively charged oxygen vacancies $\left(\mathrm{V}_{\mathrm{O}}^{-}\right.$with $S=1 / 2$ and $\mathrm{V}_{\mathrm{O}}^{2-}$ with $S=1$ ) should be stable, and such vacancies may be a feature of oxides with high dielectric constants. Such negative charge states bind the extra electrons only weakly and have polaronic nature. Moreover, both electron and hole polarons exist, and indeed local and delocalized forms may be close in energy, and so perhaps could contribute to the delocalization needed for connectivity. The relative stability of small and large polaron forms will be affected by image interactions when there is a nearby interface with a medium with a different dielectric constant. The large polaron gains a modest extra stability near an interface with the vacuum, for instance. The main problem with this polaron suggestion is that energy differences between the small and large forms vary significantly from one oxide to another. It is hard to see this as a general mechanism.

What diffuse states might be associated with surfaces? One can probably rule out roles for states with electrons primarily outside the surface (cf the exciton in $\mathrm{MgO}$ Cox and Williams 1986) as a general mechanism, since magnetism is seen in oxides whose electron affinities vary from substantial and positive $\left(\mathrm{SnO}_{2}\right)$ to probably negative $(\mathrm{MgO})$. Surface step edges and kink sites show more promise to yield diffuse states. Preliminary calculations by Dr Sushko (2009) offer some evidence from hybrid DFT for interactions of relatively long range $(1.5-2 \mathrm{~nm})$ between spins at $\mathrm{Mg}$ vacancies at a (100) edge on an $\mathrm{MgO}$ surface. Nor should one forget the early ideas of Seitz (1954) and Mott and Gurney (1940) in the context of electrons trapped by steps at interfaces. Seitz observed that the effective charge binding an electron may be less than the nominal charge. He pointed out that a kink on a step on an alkali halide crystal would have a half-integral effective charge, even though each ion has integral charge. In essence, his argument is that the removal of one ion, say $+|\mathrm{e}|$, changes a kink charge from $+Q|\mathrm{e}|$ to $-Q|\mathrm{e}|$, i.e. a change of $2 Q|\mathrm{e}|$, so $Q$ is $1 / 2$. For oxides, the comparable argument for $\mathrm{MgO}$ without trapped electrons gives effective kink charges of $\pm|\mathrm{e}|$ on 100 surfaces; for $\mathrm{SnO}_{2}$ the charges would be 0 or $\pm 2|\mathrm{e}|$. Reducing 
an effective charge reduces binding and, to some extent, gives some delocalization. Possibly more important is the fact that there will be kinks with charges of both signs. Whereas any centre with a $1 / r$ long-range potential has an infinite number of bound states (Mott and Gurney 1940; see Stoneham 1975 chapter 9), a dipole can have a significantly delocalized wavefunction, and indeed a simple dipole needs to be bigger than a threshold value to bind at all. Thus, in $\mathrm{MgO}$, the dipolar $F_{c}^{+}$centre is significantly less bound than the $\mathrm{F}^{+}$centre, for example (Henderson et al 1969). Clearly, ideas of a role for surface kinks needs a proper theory, but perhaps gains some support from the distinct but related observation of magnetic (antiferromagnetic) ordering of paramagnetic defects on partly hydroxylated $\mathrm{MgO}$ nanocrystal surfaces (Moreira et al 2008). In certain cases (Meulenberg et al 2009) chemical groups stuck to quantum dot edges lead to magnetism, though it is not clear how widespread this phenomenon is. Perhaps more important is the implication that modelling of oxide interfaces should be able to cope with delocalized states.

What diffuse states might be associated with internal interfaces or line defects? There are experimental data for nanocrystalline oxides but, apart from surface defects, are not especially informative. It is therefore necessary to look at stateof-the-art calculations. I am indebted to Professor A L Shluger, Dr K McKenna and Dr P Sushko for several points arising from their own calculations. They find that paramagnetic vacancy systems are more common than usually thought in oxides. Grain boundaries in MgO (McKenna and Shluger 2008) do show extended states just above the valence band, as well as a higher energy band associated with image states, and these states have some of the character needed. One might think of two $\mathrm{V}^{0}$ centres, each within a few interatomic spacings of a grain boundary, but perhaps $2 \mathrm{~nm}$ apart from each other. The hole wavefunctions of each $\mathrm{V}^{0}$ centre would have a small admixture of delocalized states, and these might then provide a longer-range interaction. In other words, the proximity of localized magnetic centres to an extended defect might enable a useful long-range interaction. Vacancies are predicted to segregate to grain boundaries both in $\mathrm{MgO}$ and $\mathrm{HfO}_{2}$ and, in these systems, electrons and holes can form delocalized but confined states (McKenna et al 2007). As noted above, other interfaces show interesting features, e.g. the magnetism associated with the $\mathrm{SrTiO}_{3} / \mathrm{LaAlO}_{3}$ interface (Brinkman et al 2007); this particular interface is remarkable, even showing interface superconductivity. Again, vacancies appear to play a significant role. All this is suggestive, but are there enough grain boundaries or dislocations in the nanocrystals? That is not clear. In bulk oxides, the numbers of dislocations (typically $10^{5} \mathrm{~cm} \mathrm{~cm}^{-3}$ ) and grain boundaries (typical grain sizes of $10 \mu \mathrm{m})$ are not sufficient. However, there are indications that significantly higher dislocation densities may occur in nanocrystalline oxides (Chen et al 2009).

In a technology context, the next obvious question is whether one can create or control the extended states that somehow resolve the connectivity problem? If so, the optimist might hope to write nanoscale ferromagnetic regions in oxides. There are some reasons to encourage optimism. In thin $\mathrm{HfO}_{2}$ gate oxides, the negatively charged states of oxygen vacancies can serve as percolation paths for oxide breakdown (McKenna 2009). The idea of percolation paths in the resistive switching of oxides is long established (Dearnaley et al 1970), the paths (or filaments) being formed from linked point defects. Similar ideas have been discussed for breakdown of silicon dioxide gate dielectrics. Many thin film oxides show switching between high and low resistance states, behaviour that can depend on the nature of the electrode. Such systems were comprehensively reviewed by Dearnaley et al (1970), with many recent studies surveyed by Sawa (2008), Waser and Aono (2007) and Waser et al (2009). It is natural to conjecture a connection between the two unusual phenomena, resistive switching and magnetism, in simple oxides. Some oxides do indeed show both switching and magnetism (e.g. without intentional doping $\mathrm{Al}_{2} \mathrm{O}_{3}, \mathrm{HfO}_{2}$ and $\mathrm{TiO}_{2}$; with a small transition metal doping $\mathrm{SnO}_{2}$ and $\mathrm{SrTiO}_{3}$; there may be other cases). Since it is probable that the raised conductivity (low resistance state) is associated with current flow along filaments that involve defects (Dearnaley et al 1970), are there extended states associated with these filaments? And could the diffuse states provide the connectivity between the localized paramagnetic centres needed for ferromagnetism? Very recently, Wu et al (2009) have indeed reported a correlation of magnetism with switching in $\mathrm{Mn}$-doped $\mathrm{TiO}_{2}$, finding robust ferromagnetism associated with the low resistance state. My proposal would imply that one could also write ferromagnetic behaviour even in sp oxides that show forming, and that this should be possible especially effectively at the nanoscale. What also encourages this conjecture is the fact that there is one system that can be switched, and indeed written at the nanoscale, namely $\mathrm{LaAlO}_{3} / \mathrm{SrTiO}_{3}$ (Cen et al 2009). Many studies show extended states to be clearly evident at $\mathrm{LaAlO}_{3} / \mathrm{SrTiO}_{3}$ interfaces, where a two-dimensional electron gas can form.

What about the carbons? Magnetism in carbons-whether simply carbon in its several forms, or carbon with hydrogen or other low atomic number species-has been quoted in nanodiamond (Talapatra et al 2005), irradiated graphite (Coey and Sanvito 2004, Esquinazi et al 2003), carbon nanotubes (Andriotis et al 2006), zig-zags in nanotubes (Coey and Sanvito 2004), $\mathrm{C}_{60}$ systems (Andriotis et al 2006, Mathew et al 2007) and organic molecules (McConnell 1967). The list just given is certainly incomplete, and may well be controversial. Again, as for oxides, theory gives magnetic ground states for some localized defect systems (e.g. Ma et al 2005), and several suggestions of mechanisms discussed above appear to apply. So it is credible that localized centres in carbons may be magnetic. What about the connectivity? For some carbons, at least, delocalized electronic states are more common. The electron-lattice coupling is usually far less than in oxides. Delocalized states are found even in amorphous carbon (Khan et al 2001). Ferromagnetism in carbons may be easier to explain than that of oxides.

\section{Conclusions}

Why would we want ferromagnetic oxides when there are transition metals like iron? Reasons might include robustness, or system compatibility. Thus $\mathrm{MgO}$, for instance, is stable 
in composition, not especially reactive, and maintains its properties up to high temperatures, whereas iron rusts and causes problems when it gets into silicon. These reasons are not yet compelling, but the thin film and nanocrystal results are promising, and the needs of nanoscience may make their magnetic properties desirable. If the conjecture proves right that it is possible to write the connecting extended states in some way, this might prove a route to interesting technology.

From these several strands, three points can be made. First, experiment and theory agree that many oxides, even those without transition metals, contain simple intrinsic defects that have spin triplet states occupied at room temperature. This is independent of whether or not this triplet state is the ground state, which is convenient because the sign of the singlet-triplet splitting involves a delicate balance between two energy terms. Second, spin resonance shows these simple intrinsic defects to be compact, so direct interactions between them at the measured concentrations seem insufficient for the ferromagnetism that is reasonably well documented. However, ferromagnetism might result if there were a weak admixture of some delocalized states, at least in nanocrystals or thin films. Just what these delocalized states are is uncertain, but several conjectures can be made. Since the phenomenon is common in nanocrystalline oxides, any defect system should be robust and able to be effective over a wide range of oxides, carbons and some other systems. Third, these conjectures hint at links of ferromagnetism to other novel oxide behaviours, including high carrier mobilities near interfaces and switching between low and high resistance states. This raises the possibility that magnetic behaviour, as well as conductance, might be written onto simple oxide surfaces with an atomic force microscope. These ideas need careful work to be fully credible, but imply that simple oxides are far from simple.

I have tried to separate the observational facts from conjectures. In the context of electronic structure theory, the conjectures point to significant problem areas. Understanding the magnetic behaviour seems to demand three features that test most standard electronic structure theories: the selftrapping phenomenon leading to localization of charge, the modelling of weakly bound and relatively delocalized states, and the relative energies of different spin states in which several electron spins are weakly coupled. Individually, these features are common. Together, one can say these unusual magnetic systems present an interesting combination of challenges.

\section{Acknowledgments}

This viewpoint is based on a talk at a conference celebrating the 65th birthday of Professor Michael Gillan, and I should like to thank him for insights and discussions over many years. In particular, his innovative work on oxides suggested the topic of this viewpoint to me. I should also like to thank a number of others for correspondence and discussions, without wishing to imply that they share my views. In particular, I should like to thank A N Andriotis, J M D Coey, P Esquinazi, B Henderson, K P McKenna, A L Shluger, P Sushko and A S Sundaresan for discussions, insights and telling me of work in progress.

\section{References}

Abraham D W, Frank M M and Guha S 2005 Appl. Phys. Lett. 87252502

Abraham M M, Chen Y, Boatner L A and Reynolds R W 1975 Solid State Commun. 16 1209-13

Andriotis A N, Sheetz R M and Menon M 2006 Phys. Rev. B 74153403

Brinkman A, Huijben M, van Zalk M, Huijben J, Zeitler U, Maan J C, van der Wiel W G, Rijnders G, Blank D H A and Hilgenkamp H 2007 Nat. Mater. 7493

Catlow C R A and Stoneham A M 1983 J. Phys. C: Solid State Phys. $164321-38$

Chen B, Zhang H, Dunphy-Guzman K A, Spagnoli D, Kruger M B, Muthu D V S, Kunz M, Fakra S, Hu J Z, Guo Q Z and Banfield J F 2009 Phys. Rev. B 79125406

Cen C, Thiel S, Mannhart J and Levy J 2009 Science 3231026

Coey J M D and Chambers S A 2008 MRS Bull. 331053

Coey J M D and Sanvito S 2004 Magnetism of carbon Phys. World (November) 33

Coey J M D, Wongsaprom K, Alaria J and Venkatesan M 2008 J. Phys. D: Appl. Phys. 41134012

Coey J M D, Venkatesan M and Fitzgerald C B 2005 Nat. Mater. 4173

Cox P A and Williams A A 1986 Surf. Sci. 175 L782

Cox R T 1966 Proc. XIV Colloque Ampere (Ljubljana) ed R Blinc (Amsterdam: North-Holland) p 279

Cox R T 1971 Solid State Commun. 91989

Cox R T 1972 Thesis University of Grenoble 306-7

Dearnaley G, Stoneham A M and Morgan D V 1970 Rep. Prog. Phys. 331129

Esquinazi P, Spemann D, Höhne R, Setzer A, Han K-H and Butz T 2003 Phys. Rev. Lett. 91227201

Galland D and Herv'e A 1970 Phys. Lett. A 33 1-2

Harding J H and Stoneham A M 1982 J. Phys. C: Solid State Phys. 15 4649-59

Henderson B 2006 private communication

Henderson B, To K C and Stoneham A M 1969 Phys. Rev. 1811237

Henderson B and Tomlinson A C 1969 J. Phys. Chem. Solids 301801

Hong N H, Poirot N and Sakai J 2006 Appl. Phys. Lett. 89042503

Hu J F, Zhang Z, Zhao M, Qin H W and Jiang M H 2008 Appl. Phys. Lett. 93192503

Itoh N and Stoneham A M 2001 Materials Modification by Electronic Excitation (Cambridge: Cambridge University Press)

Jaffe J E, Droubay T C and Chambers S A 2005 J. Appl. Phys. 97073908

Khan R U A, Carey J D, Silva S R P, Jones B J and Barklie R C 2001 Phys. Rev. B 63121201

Kollmar C and Klein O 1993 Acc. Chem. Res. 26 259-65

Liu Y, Lockman Z, Azizan A and MacManus-Driscoll J 2008 J. Phys.: Condens. Matter 20165201

Ma Y, Lehtinen P O, Foster A S and Nieminen R M 2005 Phys. Rev. B 72085451

Maffeo B, Herv'e A and Cox R T 1970 Solid State Commun. 8 2169-72

Mathew S, Satpati B, Joseph B, Dev B N, Nirmala R, Malik S K and Kesavamoorthy R 2007 Phys. Rev. B 75074426

McConnell H M 1967 Proc. Robert A Welch Found Conf. Chem. Res. vol 11 p 144

McKenna K P 2009 unpublished

McKenna K P and Shluger A L 2008 Nat. Mater. 7859

McKenna K P, Sushko P V and Shluger A L 2007 J. Am. Chem. Soc. 1298600

Meulenberg R W, Lee J R I, McCall S K, Hanif K M, Haskel D, Lang J C, Terminello L J and van Buuren T $2009 \mathrm{~J}$. Am. Chem. Soc. 131 6888-9 
Moreira I de P R, Wojdeł J C, Illas F, Chiesa M and Giamello E 2008 Chem. Phys. Lett. 462 78-83

Mott N F and Gurney R W 1940 Electronic Processes in Ionic Crystals (Oxford: Oxford University Press)

Muñoz Ramo D, Gavartin J L, Shluger A L and Bersuker G 2007 Phys. Rev. B 75205336

Nelson R L, Hale J W, Harmsworth B J and Tench A J 1968 Trans. Faraday Soc. 642521

Nelson R L and Tench A J 1967 Trans. Faraday Soc. 633039

Norgett M J, Pathak A P and Stoneham A M 1977 J. Phys. C: Solid State Phys. 10555

Osorio-Guill'en J, Lany S, Barabash S V and Zunger A 2006 Phys. Rev. Lett. 96107203

Pathak A P, Bartram R H and Stoneham A M 1976 J. Phys. C: Solid State Phys. $973-80$

Pemmajaru C D and Sanvito S 2005 Phys. Rev. Lett. 94217205

Raebiger H, Lany S and Zunger A 2009 Phys. Rev. B 79165202

Ramachandra Rao M S, Kundaliya D C, Ogale S B, Fu L F, Welz S J, Browning N D, Zaitsev V, Varughese B, Cardoso A, Curtin A, Dhar S, Shinde S R and Vasiliu-Doloc T 1999 Phys. Rev. Lett. 834393

Rius G and Cox R 1974 private communication

Sawa A 2008 Mater. Today 1128

Schirmer O F 2006 J. Phys.: Condens. Matter 18 R667-704

Schirmer O F, Koidl P and Reik H G 1974 Phys. Status Solidi b 62385

Seitz F 1954 Rev. Mod. Phys. 267
Stoneham A M 1975 Theory of Defects in Solids (Oxford: Oxford University Press)

Stoneham A M and Bullough R 1970 J. Phys. C: Solid State Phys. 3 L195

Stoneham A M, Gavartin J, Shluger A L, Kimmel A V, Muñoz Ramo D, Rønnow H M, Aeppli G and Renner C 2007 J. Phys.: Condens. Matter 19255208

Sundaresan A, Bhargavi R, Rangarajan N, Siddesh U and Rao C N R 2006 Phys. Rev. B 74161306

Sundaresan A and Rao C N R 2009 Nano Today 496

Sushko P 2009 private communication

Talapatra S, Ganesan P G, Kim T, Vajtai R, Huang M, Shima M, Ramanath G, Srivastava D, Deevi S C and Ajayan P M 2005 Phys. Rev. Lett. 95097201

Venkatesan M, Fitzgerald C B and Coey J M D 2004 Nature 430630

Waser R and Aono M 2007 Nat. Mater. 6 833-40

Waser R, Dittmann R, Staikov G and Szot K 2009 Adv. Mater. 212632

Wertz J E, Auzins P, Griffiths J H E and Orton J W 1959 Discuss. Faraday Soc. $\mathbf{2 8} 136$

Wu S X, Li X Y, Xing X J, Hu P, Yu Y P and Li S W 2009 Appl. Phys. Lett. 94253504

Ye X J, Song H A, Zhong W, Xu M H, Qi X S, Jin C Q, Yang Z X, Au C T and Du Y W 2008 J. Phys. D: Appl. Phys. 41155005

Zhang Z, Jeng S-P and Henrich V E 1991 Phys. Rev. B 4312004

Zhou S, Xu Q, Potzger K, Talut G, Grötzschel R, Fassbender J, Vinnichenko M, Grenzer J, Helm M, Hochmuth H, Lorenz M, Grundmann M and Schmidt H 2008 Appl. Phys. Lett. 93232507 